\title{
Spin-Excitons in Heavy-fermion Semimetals
}

\author{
Peter S Riseborough ${ }^{1}$ and S.G. Magalhaes ${ }^{2}$ \\ ${ }^{1}$ Temple University, Philadelphia, USA \\ ${ }^{2}$ Univ. Federale, Fluminense, Niteroi, Rio de Janeiro, Brazil
}

June 24, 2015

\begin{abstract}
Spin-excitons are sharp and dispersive magnetic fluctuations in paramagnetic semiconductors where the dispersion relation lies within the semiconducting gap. Spin-excitons are found in the vicinity of magnetic quantum critical points in semiconductors, much the same as antiparamagnons are precursor fluctuations for quantum critical points in metals. Here we show that this concept of spin-exciton excitations can be extended to heavy-fermion semimetals and provides a natural explanation of the magnetic modes found by inelastic neutron scattering experiments on paramagnetic $\mathrm{CeFe}_{2} \mathrm{Al}_{10}$.
\end{abstract}

Keywords: Kondo Insulators, Kondo Semimetals, $\mathrm{CeFe}_{2} \mathrm{Al}_{10}$, Spin-excitons, Quantum Critical Point

Corresponding Author: prisebor@temple.edu

\section{Introduction}

Heavy-fermions metals are frequently found in the vicinity of quantum critical points, and can be phenomenologically described by the Doniach phase diagram [1]. The Doniach phase diagram is not a phase diagram but instead is a statement about the relative energy scales of a lattice of magnetic ions which interact with the conduction electrons via a Kondo exchange interaction $J_{K}$. For an isolated impurity, the Kondo temperature is given by

$$
k_{B} T_{K} \sim \rho^{-1} \exp \left[-\frac{1}{N \rho J_{K}}\right],
$$

where $\rho$ is the conduction band density of states and $N$ is the degeneracy of the local moment $(N \geq 2)$. For temperatures below the Kondo temperature, an isolated local moment will be compensated by the magnetization of a screening cloud of conduction electron leading to a singlet state. The Kondo effect competes with magnetic ordering promoted by an RKKY-like interaction which is second-order in the Kondo interaction. The RKKY-like interaction between two local moments has a magnitude of $\rho J_{K}^{2}$ [2]. Comparison of the two energy scales 
suggests that, for the smallest values of $\rho J_{K}$, a magnetic phase will be stable. However, for $N \geq 2$, a non-magnetic state will result at larger $\rho J_{K}$. A quantum critical point will separate the two phases. Since heavy-fermion systems are phenomenologically characterized by exceptionally small Kondo temperatures $1 \gg \rho T_{K}$, one infers that heavy-fermion materials are to be found near quantum critical points.

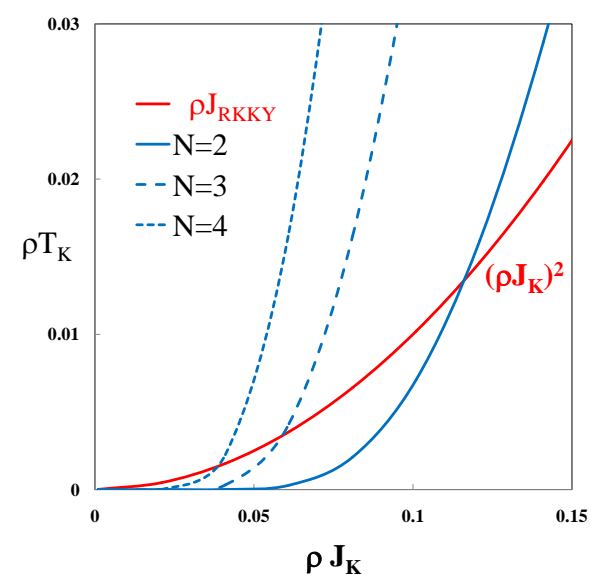

Figure 1: The Doniach diagram in which the dimensionless RKKY interaction $\left(\rho J_{K}\right)^{2}$ and the dimensionless Kondo temperature $\rho T_{K}$ is plotted as a function of the Kondo exchange interaction $\rho J_{K}$, for various values of the degeneracy $N$.

The conventional description of a quantum critical point in a metal $[3,4,5,6]$ is that of a second-order phase transition, at $T=0$, which is dominated by overdamped, low-frequency, large-amplitude magnetic fluctuations. These types of fluctuations were first identified within the Random Phase Approximation and are known as either paramagnons $[7,8]$ or anti-paramagnons $[9,10]$, respectively, depending on whether the paramagnetic state is unstable to either a ferromagnetic or antiferromagnetic state. These fluctuations are over-damped as they can decay into particle-hole pairs, but nevertheless, their frequencies soften as the wavevector of the fluctuations approaches a wavevector $Q$ which characterizes the magnetically ordered state. As the quantum critical point is approached, by either varying pressure or by decreasing temperature, the frequencies of the fluctuations decrease and their amplitudes increase.

Spin-excitons are magnetic fluctuations that are found in semiconductors that are close to a quantum critical point and are analogous to the paramagnons or anti-paramagnons found in metals. A spin-exciton has a sharp dispersion relation for frequencies smaller than a characteristic energy gap since, unlike damped antiparamagnons in metals, there are no low-frequency particlehole channels available in a semiconductor. The minimum in the spin-exciton's dispersion relation occurs at a wave-vector corresponding to the wavevector $Q$ of the magnetically ordered state and is finite but softens as the quantum critical 
point is approached.

Spin-excitons were predicted to occur [11] in the heavy-fermion semiconductor $\mathrm{Ce}_{3} \mathrm{Bi}_{4} \mathrm{Pt}_{3}$ [12], but were first observed in $\mathrm{SmB}_{6}[13,14,15]$ and later in $\mathrm{YbB}_{12}[16,17]$. Since the excitation is not seen in optical absorption experiments, the excitations are purely magnetic and are not charged excitations [18]. The spin-excitons were first described within the framework of the Periodic Anderson Model in the strong-coupling limit where the Coulomb correlations are treated using the slave boson method [19, 20, 21]. In that treatment, the quasi-particles are described by renormalized bands to order $1 / N$ by the mean-field approximation [19]. The magnetic exchange interaction between the quasi-particles are mediated by the exchange of slave bosons [2], and is of the order of $\frac{1}{N^{2}}$.

Recently, spin-excitons were observed in the material $\mathrm{CeFe}_{2} \mathrm{Al}_{10}[22,23]$ which, unlike the heavy-fermion semiconductors, only shows a pseudogap in the density of states. Therefore, like CeNiSn [24, 25, 26], this material is a member of the class of heavy-fermion semimetals. The relation of the nesting vector in $\mathrm{CeFe}_{2} \mathrm{Al}_{10}$ to the ordering wavevector in the related antiferromagnetic compound $\mathrm{CeOs}_{2} \mathrm{Al}_{10}$ strengthens the identification of the spin-exciton as a precursor magnetic fluctuation. In this manuscript, we shall describe the theory of the spin-exciton and show how the theory can be extended to heavy-fermion semimetals.

\section{The Model and Calculation}

The spin-exciton can be derived as a magnetic excitation of the paramagnetic state of the Anderson Lattice Model by using the Random Phase Approximation. The Hamiltonian of the Anderson Lattice Model is given by

$$
\hat{H}=\hat{H}_{f}+\hat{H}_{d}+\hat{H}_{f d},
$$

where $\hat{H}_{f}$ describes the felectron states, $\hat{H}_{d}$ describes the conduction electron states and $\hat{H}_{f d}$ describes the hybridization which couples the $\mathrm{f}$ and d states. The Hamiltonian $\hat{H}_{f}$ is given by

$$
\hat{H}_{f}=\sum_{\underline{k}, \sigma} E_{f}(\underline{k}) f_{\underline{k}, \sigma}^{\dagger} f_{\underline{k}, \sigma}+\sum_{i} U f_{i, \uparrow}^{\dagger} f_{i, \downarrow}^{\dagger} f_{i, \downarrow} f_{i, \uparrow},
$$

where the operators $f_{k, \sigma}^{\dagger}$ and $f_{i, \sigma}^{\dagger}$ create electrons of spin $\sigma$, respectively, a Bloch state with Bloch wavevector $\underline{k}$ and in a Wannier state at site $\underline{R}_{i}$. The first term represents a relatively flat $\mathrm{f}$ band, and the second term represents a highlyscreened Coulomb interaction between the f electrons on the same lattice site. The Hamiltonian $\hat{H}_{d}$ describing tight-binding conduction band states is given by

$$
\hat{H}_{d}=\sum_{\underline{k}, \sigma} \epsilon(\underline{k}) d_{\underline{k}, \sigma}^{\dagger} d_{\underline{k}, \sigma},
$$


where $d_{k, \sigma}^{\dagger}$ and $d_{\underline{k}, \sigma}$, respectively, create or annihilate an electron of spin $\sigma$ in the conduction band state labeled by Bloch wavevector $\underline{k}$. The hybridization is represented by

$$
\hat{H}_{f d}=\sum_{\underline{k}, \sigma}\left[V(\underline{k}) f_{\underline{k}, \sigma}^{\dagger} d_{\underline{k}, \sigma}+V^{*}(\underline{k}) d_{\underline{k}, \sigma}^{\dagger} f_{\underline{k}, \sigma}\right],
$$

where the first term represents a process where an electron hops from the conduction band into the $\mathrm{f}$ band and the second term represents the inverse process. In what follows, we shall use a $\underline{k}$-independent hybridization $V=0.5 t, E_{f}=0$ and with a tight-binding conduction band of width $6 t$.
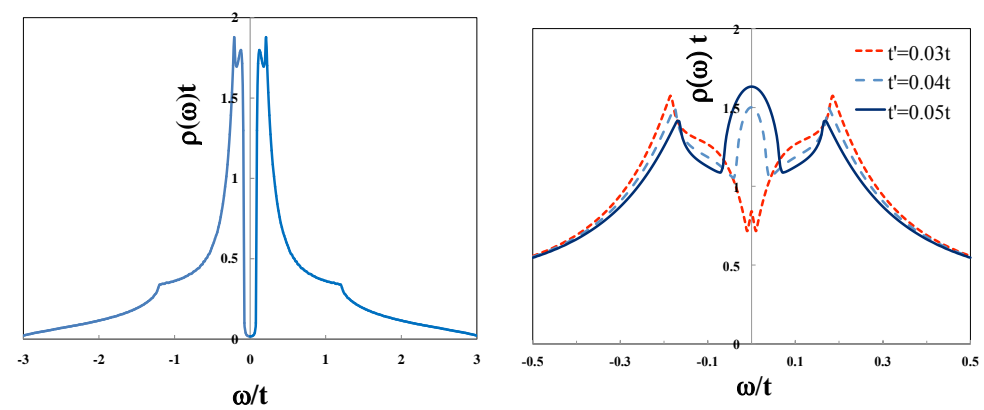

Figure 2: (Left Panel) The total density of states of the semiconducting Anderson Lattice Model, with a flat $f$ band $t^{\prime}=0$. (Right Panel) The total density of states for the semimetallic case of the Anderson Lattice Model with a slightly dispersive $\mathrm{f}$ band, where the $\mathrm{f}-\mathrm{f}$ hopping matrix elements are chosen as $t^{\prime}=0.03 t$, $t^{\prime}=0.04 t$ and $t^{\prime}=0.05 t$.

Quasi-particle bands for the paramagnetic state can be obtained within either standard mean-field theory or by using slave boson mean-field theory, to order $\frac{1}{N}$. In both cases, the quasiparticle density of states shows a hybridization gap which is proportional to the squared modulus of the hybridization matrix elements. The quasiparticle dispersion relations $E^{ \pm}(\underline{k})$ of the hybridized bands are given by

$$
E^{ \pm}(\underline{k})=\left(\frac{E_{f}(\underline{k})+\epsilon(\underline{k})}{2}\right) \pm \sqrt{\left(\frac{E_{f}(\underline{k})-\epsilon(\underline{k})}{2}\right)^{2}+|V(\underline{k})|^{2}} .
$$

The factors $A^{ \pm}(\underline{k})$ that project onto the f characters of the quasiparticle bands are given by

$$
\left|A^{ \pm}(\underline{k})\right|^{2}=\frac{1}{2}\left[1 \pm \frac{E_{f}(\underline{k})-\epsilon(\underline{k})}{\sqrt{\left(E_{f}(\underline{k})-\epsilon(\underline{k})\right)^{2}+|V(\underline{k})|^{2}}}\right] .
$$

In the slave boson mean-field theory, the gap is reduced by a factor of $\left[1-n_{f}(T)\right]$ and represents the Kondo condensation energy of the lattice. The bare $f$ level is 
also shifted towards the chemical potential. The total density of states is shown in fig.(2).

The quasiparticles are subjected to a residual interaction $J$. In the slave boson theory, the interaction of order $\frac{1}{N^{2}}$ is mediated by the exchange of two slave bosons, as has been described by Doniach [2]. For the usual mean-field theory, $J$ becomes identical to $U$ and is independent of momentum transfer. The magnetic susceptibility of the f electrons can be evaluated in the Random Phase Approximation, leading to the following result

$$
\chi(\underline{q}, \omega)=\frac{\chi^{(0)}(\underline{q}, \omega)}{1-J \chi^{(0)}(\underline{q}, \omega)},
$$

where $\chi^{(0)}(q, \omega)$ is the quasi-particle susceptibility as obtained in mean-field theory. The mean-field Lindhard susceptibility is given by

$\chi^{(0)}(\underline{q}, \omega)=\frac{1}{N} \sum_{\underline{k}, \pm, \mp}\left|A^{ \pm}(\underline{k}+q)\right|^{2}\left|A^{\mp}(\underline{k})\right|^{2}\left[\frac{f\left(E^{ \pm}(\underline{k}+\underline{q})\right)-f\left(E^{\mp}(\underline{k})\right)}{\omega-E^{ \pm}(\underline{k}+\underline{q})+E^{\mp}(\underline{k})}\right]$.

Semiconducting Case

The density of states for the semiconducting state is shown in the left panel of fig.(2). The density of states has a gap and, if the chemical potential $\mu$ is placed within the gap, the model describes a semiconductor.
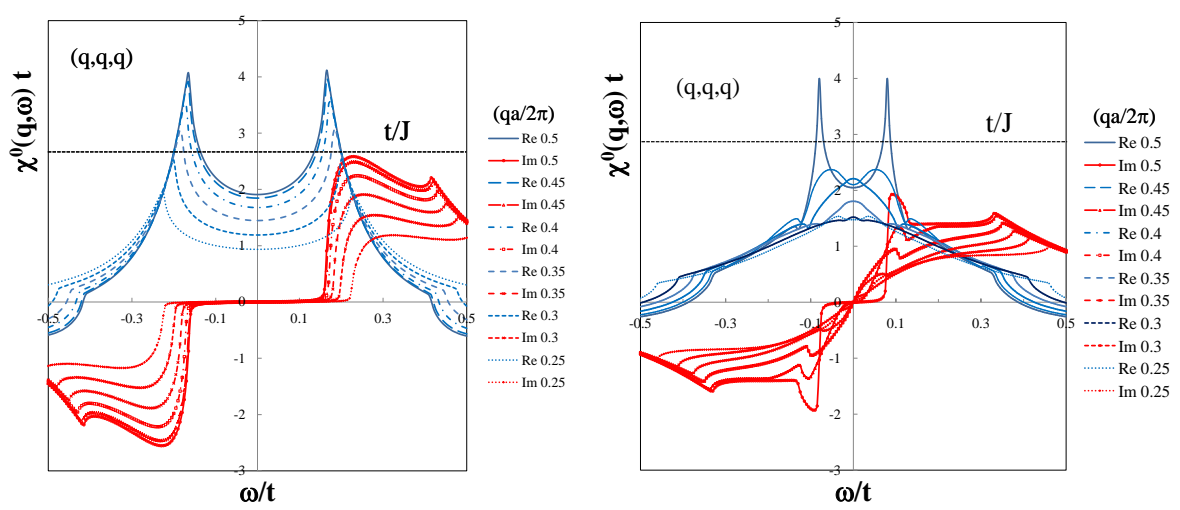

Figure 3: The dimensionless real (blue lines) and imaginary (red decorated lines) parts of the dynamic quasi-particle susceptibility $\chi^{(0)}(\underline{q}, \omega)$ of the Anderson Lattice Model for various wavevectors $\underline{q}$. The wavevector $\underline{q}$ is directed along the body-diagonal and the $q$ values are shown in the legend. (Left Panel) The semiconducting case where $t^{\prime}=0$ and the chemical potential $\mu$ is within the hybridization gap. (Right Panel) The semimetallic case with $t^{\prime}=0.05 t$, where the chemical potential is placed within the pseudogap, $\mu=0.04 t$. 


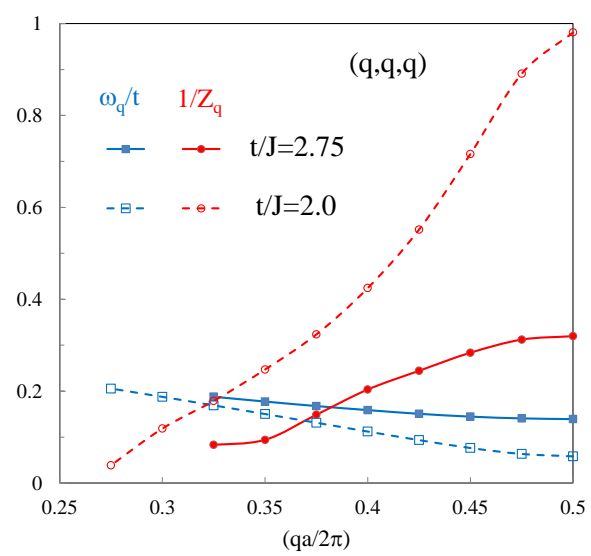

Figure 4: The intensity $Z_{q}^{-1}$ and dispersion relation $\omega_{q} / t$ of the spin-exciton for two different values of $J / t$. As $J$ increases, the spin-exciton dispersion softens more and its intensity increases.

For sufficiently large values of $J$, the denominator of the susceptibility may vanish at a frequency $\omega_{q}$ within the gap

$$
1-J \Re e \chi^{(0)}\left(\underline{q}, \omega_{q}\right)=0
$$

and

$$
\Im m \chi^{(0)}\left(\underline{q}, \omega_{q}\right)=0 .
$$

At this frequency, the density of magnetic excitations reduces to a delta function peak

$$
\begin{aligned}
\frac{1}{\pi} \Im m \chi(\underline{q}, \omega) & =\frac{1}{J} \delta\left(1-J \Re e \chi^{(0)}(\underline{q}, \omega)\right) \\
& =\left[\left.J^{2} \frac{\partial}{\partial \omega} \Re e \chi^{(0)}(\underline{q}, \omega)\right|_{\omega=\omega_{q}}\right]^{-1} \delta\left(\omega-\omega_{q}\right)
\end{aligned}
$$

The spin-exciton has zero width as there is no available channel for decay into electron-hole pairs. The dependence of the intensity and frequency on the wavevectors $q$ along the body-diagonal are shown in fig.(4). It is seen that the dispersion relation shows a minimum at the vertex of the Brillouin zone at which the intensity attains its maximum value. The relation between the intensity and frequency follows from the local minimum in the real part of the mean-field susceptibility at $\omega=0$. As $\underline{q}$ is varied away from $(1,1,1)$, the excitation energy increases and eventually enters the continuum where the mode rapidly broadens and exists as a resonance.

Semimetallic Case 


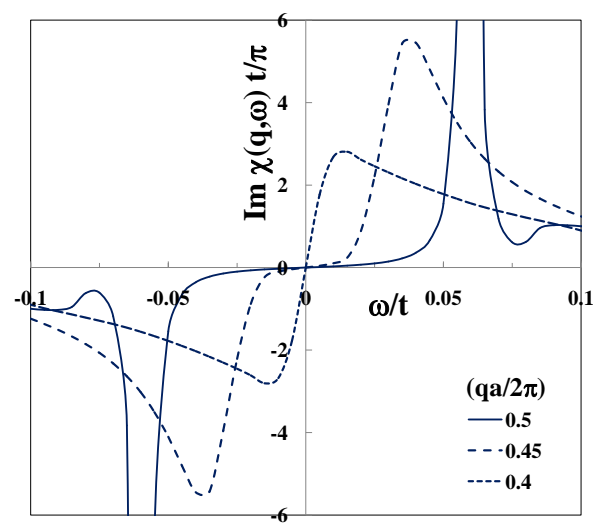

Figure 5: The magnetic spectral density for the semi-metallic case, for the same set of parameters used in fig.( $3 \mathrm{~b})$ and three different $q$ values. The spectrum resembles a delta function at $\frac{q a}{2 \pi}=0.5$ with weight 0.1 while for $\frac{q a}{2 \pi}=0.45$ the spectrum exhibits a lower threshold and the weight has increased to 0.3. The wave vector $\frac{q a}{2 \pi}=0.4$ corresponds to an incommensurate quantum critical point and the spectrum has the usual quasi-elastic form.

The above theory can be easily extended to the case of heavy-fermion semimetals (such as CeNiSn [24, 25]) in which the density of states no longer has a gap, but instead, has a pseudogap. As pointed out by Ikeda and Miyake [27], the pseudogap in CeNiSn can be produced by having nodes in the hybridization matrix elements. Alternatively, a pseudogap in the density of states can be produced by introducing a slight dispersion in the $\mathrm{f}$ band. The density of states for various values of the f-band hopping strength $t^{\prime}$ is shown in the right panel of fig.(2). It is seen that as $t^{\prime}$ increases, the total DOS still has a pseudogap, and a small peak also develops at the center of the gap representing the growth of small electron and hole pockets. The integrated intensity of the peak provides a measure of the Fermi-surface radii. This form of the density of states has been inferred from measurements of the resistivity and specific heat on CeNiSn $[25,26]$. The mean-field dynamic susceptibility $\chi^{(0)}(\underline{q}, \omega)$ shows nesting near $(1,1,1)$ between the shallow electron and hole pockets, as seen in the right panel of fig.(3). It is seen that a similar value of $J$ can produce a fairly sharp spin-exciton in the semimetallic case similar to the semiconducting case. As is also seen for sufficiently large $t^{\prime}$, the paramagnetic state eventually gives way to commensurate/incommensurate magnetic ordering and real Goldstone modes are formed.

\section{Conclusions}

In summary, we have shown that the sharp, dispersive spin-exciton excitations found within the gap of paramagnetic heavy-fermion semiconductors have their 
analogues in paramagnetic heavy-fermion semimetals due to the nesting of shallow electron and hole pockets. The excitations consist of relatively sharp excitations that disperse in the pseudogap having a minimum excitation energy and maximum intensity at the wavevector associated with a nearby quantum critical point. This theory may describe the magnetic excitations recently found $[22,23]$ in paramagnetic $\mathrm{CeFe}_{2} \mathrm{Al}_{10}$ by inelastic neutron scattering experiments.

\section{Acknowledgements}

The work at Temple was supported by an award from the US Department of Energy, Office of Basic Energy Sciences, Material Science via the award DEFG02-01ER45872.

\section{References}

[1] S. Doniach, Physica B \& C, 91, 231-234 (1977).

[2] S. Doniach, Phys. Rev. B, 35, 1814-1821 (1987).

[3] T. Moriya, J. Mag. Mag. Mat. 14, 1-46 (1979).

[4] J.A. Hertz, Phys. Rev. B, 14, 1165-1184 (1976).

[5] A.J. Millis, Phys. Rev. B, 48, 7183-7196 (1993).

[6] M.A. Continentino, Phys. Reps. 239, 179-213 (1994).

[7] S. Doniach and S. Engelsberg, Phys. Rev. Lett. 17, 750 (1966).

[8] N.F. Berk and J.R. Schrieffer, Phys. Rev. Lett. 17, 433 (1966).

[9] T. Moriya, Phys. Rev. Lett. 24, 1433-1436 (1970).

[10] H. Hasegawa and T. Moriya, J. Phys. Soc. Japan, 36, 1542-2553 (1974).

[11] P.S. Riseborough, Phys. Rev. B, 45, 13984 (1992).

[12] A. Severing, J.D. Thompson, P.C. Canfield, Z. Fisk and P.S. Riseborough) Phys. Rev. B, 44, 6832, (1991).

[13] P.A. Alekseev, J.-M. Mignot, J. Rossat-Mignod, V.N. Lazukov, I.P. Sadikov, E.S. Konalova and Y.B. Paderno, J. Phys. Cond. Mat. 7, 289 (1995).

[14] P.A. Alekseev, V.N. Lazukov, K.S. Nemovskii and I.P. Sadikov, J. Exp. Theor. Phys. 111, 285 (2010). 
[15] W.T. Fuhrman, J. Leiner, P. Nikolic, G.E. Granroth, M.B. Stone, M.D. Lumsden, L. DeBeer-Schmidt, P.A. Alekseev, J.-M. Mignot, S.M. Koopayeh, P. Cottingham, W.A. Phelam, L. Schloop, T.M. McQueen and C. Broholm, Phys. Rev. Lett. 114, 036401 (2015).

[16] A. Bouvet, T. Kasuya, M. Bonnet, L.P. Regnault, J. Rossat-Mignod, F. Iga, B. Fak and A. Severing, J. Phys. Cond. Mat. 10, 5667-5677 (1998).

[17] F. Iga, A. Bouvet, L.P. Regnault, T. Takabatake, A. Hiess and T. Kasuya, J. Phys. Chem. Solids, 60, 1193-1196 (1999).

[18] B. Gorshunov, N. Sluchanko, A. Volkov, M. Dressel, G. Knebel, A. Loidl, and S. Kunii, Phys. Rev. B, 59, 1808 (1999).

[19] P.S. Riseborough, Annalen der Physik, 9, 813-820 (2000).

[20] P.S. Riseborough, J. Mag. Mag. Mat. 226, 127-128 (2001).

[21] P.S. Riseborough, Phys. Rev. B, 68, 235213 (2003).

[22] J.M. Mignot, P.A. Alekseev, J. Robert, S. Petit, T. Nishioka, M. Matsumura, R. Kobayashi, H. Tanida, H. Nohara and M. Sera, Phys. Rev. B, 89, 161103 (2014).

[23] D.T. Adroja, A.D. Hillier, Y. Muro, J. Kajino, T. Takabatake, P. Peratheepan, A.M. Strydom, P.P. Deen, F. Demmel, J.R. Stewart, J. W. Taylor, R.I. Smith, S. Ramos and M.A. Adams, Phys. Rev. B 87, 224415 (2013).

[24] G. Nakamoto, T. Takabatake, H. Fujii, A. Minami, K. Maezawa, I. Oguro and A.A. Menovsky, J. Phys. Soc. Jpn. bf 64, 4834 (1995).

[25] K. Izawa, T. Suzuki, T. Fujita, T. Takabatake, G. Nakamoto, H. Fujii and K. Maezawa, Phys. Rev. B, 59, 2599 (1999).

[26] S. Nishigori, H. Goshima, T. Suzuki, T. Fujita, G. Nakamoto, H. Tanaka, T. Takabatake and H. Fujii, J. Phys. Soc. Jpn. 65, 2614 (1996).

[27] H. Ikeda and K. Miyake, J. Phys. Soc. Jpn. 65, 1769 (1995). 